\title{
Social security, retirement age and optimal income taxation
}

\author{
Helmuth Cremer ${ }^{\mathrm{a}, *}$, Jean-Marie Lozachmeur ${ }^{\mathrm{b}}$, Pierre Pestieau ${ }^{\mathrm{c}}$ \\ ${ }^{\text {a }}$ GREMAQ and IDEI, University of Toulouse, France \\ ${ }^{\mathrm{b}}$ GREMAQ, University of Toulouse, France \\ ${ }^{\mathrm{c}}$ CREPP, Université de Liège, CORE, Université Catholique de Louvain and Delta, France
}

Received 31 July 2002; received in revised form 26 May 2003; accepted 14 October 2003

Available online 31 January 2004

\begin{abstract}
It is often argued that implicit taxation on continued activity of elderly workers is responsible for the widely observed trend towards early retirement. In a world of laissez-faire or of first-best efficiency, there would be no such implicit taxation. The point of this paper is that, when first-best redistributive instruments are not available, because some variables are not observable, the optimal policy does imply a distortion of the retirement decision. Consequently, the inducement of early retirement may be part of the optimal tax-transfer policy. We consider a model in which individuals differ in their productivity and their capacity to work long and choose both their weekly labor supply and their age of retirement. We characterize the optimal non-linear tax-transfer that maximizes a utilitarian welfare function when weekly earnings and the length of active life are observable while individuals' productivity and health status are not observable.

(C) 2003 Elsevier B.V. All rights reserved.
\end{abstract}

JEL classification: H55; H23; E62

Keywords: Social security; Retirement age; Implicit taxation; Disability insurance

\section{Introduction}

A trend towards early retirement is currently observed in most European countries. Participation rates for men aged 60-64, which were above $70 \%$ in the early 1960 s, have fallen to $57 \%$ in Sweden and to below $20 \%$ in Belgium, France Italy and the Netherlands.

* Corresponding author. Tel.: +33-5-61-12-86-06; fax: +33-5-61-12-86-37.

E-mail address: helmut@cict.fr (H. Cremer). 
Similarly, the average labor participation in the age group 55-64 has declined and now ranges from $24 \%$ in Belgium, to $88 \%$ in Iceland, with the bulk of countries closer to Belgium than to Iceland. Early retirement per se is of course a blessing for a society, which values consumption of leisure. However, it also puts pressure on the financing of health care and pension schemes. This problem is made worse by growing longevity. In the European Union, life expectancy at age 65 has increased by more than 1 year per decade since 1950. As a consequence, instead of 45-50 years of work and 5-10 years of retirement of half a century ago, a young worker can now expect to work for 30-35 years and retire for $15-20$ years.

The effective retirement age varies across individuals and depends on features such as wealth, productivity and health. In addition, retirement decisions are likely to be affected by the pension system. When there is no pension system (utility maximizing) people retire when the marginal utility of inactivity is equal to their marginal productivity at work. People in poor health and with low productivity will retire earlier than people in good health and with high productivity. When there is a pension system, this tradeoff may or may not be affected, depending on the design of the benefit formula. In a first-best (full information) setting, an optimal retirement system would imply the same tradeoff. Such a pension system can be referred to as neutral or actuarially fair. ${ }^{1}$

In reality, pension systems are typically not neutral and they distort the retirement decision. As it has been shown by a number of authors, notably (Gruber and Wise, 1999; Blondal and Scarpetta, 1998a,b), the observed age of retirement is likely to be distorted downwards in a number of countries. The main explanation for this distortion appears to be the incentive structure implied by social protection programs aimed at elderly workers: pension plans but also unemployment insurance, disability insurance and early retirement schemes. The authors show that prolonged activity for elderly workers is subject to an implicit tax, which includes both the payroll marginal tax and forgone benefits. Consequently, social protection systems are far from being actuarially fair at the margin in countries such as Belgium, France, Germany or the Netherlands where people retire relatively early. On the other hand, in Japan, Sweden and the US the implicit tax is much lower so that the system tends to be rather neutral and people retire much later.

These results are essentially positive. Nevertheless, they are often, at least implicitly, given a normative connotation and used to advocate reforms tending to remove the bias in the benefit formulas. This raises the question of whether a bias in the benefit formula in favor of early retirement is necessarily the sign of a bad policy. We show in this paper that this implicit tax on postponed retirement is not necessarily due to bad design but can be due to the desire by public authorities of using social security for redistribution when nondistortionary tools are not available.

To address this issue, we determine in the line of Mirlees (1971) the social security benefits, payroll taxation and retirement age policy that are optimal from a utilitarian perspective. We consider a setting with heterogeneous individuals differing in two unobservable characteristics: level of productivity and health status. We study the design

\footnotetext{
1 We are concerned with actuarial fairness at the margin (no distortion) and not with global actuarial fairness (benefits are equal to contributions), which, by definition, is violated by a redistributive scheme.
} 
of a non-linear tax-transfer function depending upon two variables ${ }^{2}$ : the weekly income and the retirement age. We show that, in a setting of asymmetric information, a distortion towards early retirement is desirable for some individuals. More precisely, the optimal policy in the two-type case induces highly productive and healthy workers to retire efficiently (namely when their labor disutility is marginally equal to their productivity) while less productive and less healthy workers are induced to retire earlier. We also show that the tradeoff between weekly labor supply and retirement age (for a given lifetime income) may or may not be distorted. When a distortion is called for, its sign depends on whether the "dominant" source of heterogeneity is health or productivity. When individuals differ mainly (or exclusively) in productivity, the distortion goes against weekly labor supply. When health differentials are dominant, the distortion goes against retirement age.

The two dimensions of heterogeneity are a crucial ingredient of our analysis. When designing a redistributive social security system, it is important to take into account the wide variability in the capacity to work - a variability that is likely to widen as life expectancy increases. The practical issue is how to care for elderly workers who are in poor health without, at the same time, opening the door of retirement to those who would like to stop working but are quite capable of continuing. Consequently, a reform of social security ought to include a close connection between pensions systems and the system of disability insurance as well as the determination of a more flexible retirement age together with actuarial adjustment of yearly benefits. The ideal outcome would then be to have early retirees because of poor health receive relatively generous benefits while early retirees unwilling to continue working would receive actuarially low pensions.

There exists a theoretical literature dealing with various aspects of the issue of social security, disability insurance and retirement. It focuses on long-term labor contracts encompassing retirement rules (Lazear, 1979) and the implicit inducement to retirement of existing public and private pension plans (Crawford-Lilien, 1981; Fabel, 1994 see also Lumsdaire and Mitchell, 1999). This literature is mainly positive; it analyzes retirement behavior in order to explain the observed evolutions in retirement practice. Our paper uses a normative approach, which is intended to provide a benchmark against which the positive results can be assessed. In that respect, it is in the vein of Diamond and Mirrlees (1986) who derive disability contingent retirement rules. We shall further discuss the link between our approach and this earlier work in the concluding section. At this point, it is important to stress that we encompass income taxation, disability insurance, early retirement schemes and social security in our non-linear tax-benefit scheme. To pursue this rather ambitious endeavor, we admittedly have to simplify other aspects of the model. In particular, we essentially assume away the intertemporal aspects, which would bring in issues of uncertainty, commitment, liquidity constraints, etc. Unemployment insurance is not considered either as we assume full employment.

The rest of the paper is organized as follows. Section 2 presents the basic model while the laissez-faire and the first-best solutions are studied in Section 3. In Section 4, the second-best policy is studied. We characterize the optimal (incentive compatible) utilitarian allocation and the implementing income tax and social security benefit functions. To keep the presentation simple, we focus on an economy with two types of individuals.

\footnotetext{
2 See Maderner and Rochet (1995) who also deal with this problem in another setting.
} 
Section 5 provides some numerical examples of a three-type setting. It also considers the possibility of (costly) auditing for the health status as additional departure from the theoretical model.

\section{The model}

Most of our analysis is based on a reduced form specification. We start by presenting the underlying micro model and show how it leads to the specification we use. This detour is necessary to grasp the proper interpretation of our setting. Consider an individual who has preferences over consumption $c$ and labor $l$, which can be expressed by an instantaneous utility function $U(t)$ assumed to be additively separable:

$$
U(t)=u(c(t))-r(t) V(l(t))
$$

where $u$ and $V$ fulfill the usual assumptions and $r(\tau)$ denotes the instantaneous increasing intensity of labor disutility. Let date 0 denote entrance to the labor force, $h$, the maximum life span and $z$, the retirement age (length of working life). For simplicity, we shall often refer to $l$ as "weekly" labor supply. Though somewhat abusive, this terminology is also useful to avoid confusion with $z$, which represents another dimension of (lifetime) labor supply. With the interest rate and the discount factor both equal to 0 , lifetime utility can be written as:

$$
U=\int_{0}^{h} u(c(t)) \mathrm{d} t-\int_{0}^{z} r(t) V(l(t)) \mathrm{d} t
$$

Assuming a constant weekly productivity $w$ over time, the lifetime budget constraint is:

$$
\int_{0}^{h} c(t) \mathrm{d} t=\int_{0}^{z}[w l(t)-\tau(w l(t))] \mathrm{d} t+(h-z) p(z)
$$

where $\tau(w l(t))$ is an instantaneous non-linear tax depending on labor income and $p(z)$ the instantaneous level of pension which may depend on the individual's retirement age (via the benefit formula). The total (lifetime) retirement benefits are given by $(h-z) p(z)$. For the sake of simplicity, we impose that the choice $l(t)=l$ is time invariant. ${ }^{3}$ Separability, concavity of the instantaneous utility functions, perfect capital markets and certain lifetimes imply that each individual will set his level of consumption equal in all periods.

Denoting $y=w l$, one can rewrite the budget constraint as follows:

$$
h c=z y-T(y, z)
$$

where

$$
T(y, z)=z \tau(y)-(h-z) p(z),
$$

is the difference between total tax payments and total retirement benefits. The function $T(y, z)$ represents the net social security cum income tax paid by an individual.

\footnotetext{
3 Without such a restriction, $l(\tau)$ would be decreasing over time.
} 
Alternatively, we can think of $-T(y, z)$ as the net transfer an individual receives from the social security system. Differentiating $T$ yields the implicit tax on retirement that has estimated Gruber and Wise (1999). To see this note that

$$
\frac{\delta T(y, z)}{\delta z}=\tau(y)+p(z)-(h-z) p^{\prime}(z) .
$$

In words, an additional year of work may imply a double cost: the payroll tax $\tau(y)$ and foregone benefits if $p(z)>0$. On the other hand, postponing retirement may imply higher per period benefits during retirement. This positive effect (negative cost) is captured by the third term on the RHS of Eq. (5). In the rest of the paper, we use this reduced tax function $T(y, z)$.

With $c$ and $l$ constant over time, lifetime utility is given by

$$
U=h u(c)-V(l) R(z)
$$

where

$$
R(z)=\int_{0}^{z} r(t) \mathrm{d} t
$$

The function $R(z)$ denotes the disutility for a working life of length $z$; we have $R^{\prime}(z)=r(z)>0$ and $R^{\prime \prime}(z)=r^{\prime}(z)>0$. Labor disutility, regarding the length of working life $z$, can be interpreted in terms of an indicator of health. Healthy individuals accordingly would have a lower $R(z)$ than individuals whose poor health makes it harder to work beyond a certain age. There is another term in the labor disutility, which concerns the length of work week, $V(l)$. We assume that $V(l)$ is the same for all. Even though it may appear to be restrictive at first, this assumption in itself does not imply any major restrictions. In particular, it does not imply that all individuals have the same disutility of (weekly) work. Nor does it rule out that individuals in poor health may dislike intense work pace as much as a long working life. While this point is obscured by the reduced form, it is brought out clearly by the underlying original specifications, Eqs. (1) and (2) along with the definition (7). These expressions show that differences in $R(z)$ just reflect differences in $r(t)$ and thus in the instantaneous disutility of work $r(t) V(l(t))$. Roughly speaking the rate at which disutility of labor increases with age is larger when an individual is in poor health (his $R$ is larger) than when he is in good health. ${ }^{4}$ We shall further comment on these issues at the end of Section 3.

Each individual is characterized by two parameters: his productivity level $w_{i}$ and his disutility for the retirement age $R_{j}(z)=R\left(z ; \alpha_{j}\right)$ with $\delta R / \delta \alpha_{j}>0$. There are two levels of productivity $w_{h}$ and $w_{l}$ with $w_{h} \geq w_{l}$. Similarly, the health status parameter takes two values with $\alpha_{h} \geq \alpha_{l}$ so that $R_{h}(z) \geq R_{l}(z)$ for every $z$. Note that the subscript $h$ when associated with $\omega$ refers to the "good" (high productivity) type, while $h$ associated with $R$ is the "bad" (high disutility of remaining in the labor force) type. We denote a

\footnotetext{
4 In that sense, "health" is viewed here as a once and for all characteristic of an individual. This is one of the major differences between our model and the Diamond and Mirrlees setting; see Section 6 for further discussion.
} 
type of individual with subscripts $(i, j), i$ denoting the productivity index and $j$ the age of retirement disutility index.

\section{The laissez-faire economy and the first-best}

\subsection{The laissez faire}

In a laissez-faire economy, deleting the subscripts referring to individuals types, every agent solves the following problem:

$$
\max _{l, z} h u\left(\frac{w l z}{h}\right)-V(l) R(z)
$$

The first order conditions with respect to $l$ and $z$ are, respectively:

$$
\begin{aligned}
& u^{\prime}(c) w z-V^{\prime}(l) R(z)=0 \\
& u^{\prime}(c) w l-V(l) R^{\prime}(z)=0 .
\end{aligned}
$$

With Eqs. (9) and (10), one obtains the usual equality between marginal rates of substitution between work and consumption and the corresponding relative price:

$$
\begin{aligned}
& \operatorname{MRS}_{c l}=\frac{V^{\prime}(l) R(z)}{u^{\prime}(c)}=w z \\
& \operatorname{MRS}_{c z}=\frac{V(l) R^{\prime}(z)}{u^{\prime}(c)}=w l
\end{aligned}
$$

where $\mathrm{MRS}_{a b}$ stands for the marginal rate of substitution between $a$ and $b$. Combining Eqs. (9) and (10), the tradeoff between $l$ and $z$ is determined by:

$$
\operatorname{MRS}_{l z}=\frac{V(l) R^{\prime}(z)}{V^{\prime}(l) R(z)}=\frac{l}{z}
$$

To interpret condition (13) observe that the maximization of Eq. (8) requires the minimization of "effort" as described by the following dual problem:

$$
\begin{aligned}
& \min _{l, z} E=V(l) R(z) \\
& \text { s.t. } I=w l z,
\end{aligned}
$$

where $I$ represents lifetime earnings and $E$ denotes aggregate effort (or utility cost). Fig. 1 represents problem $(14)$ in the $(z, l)$ space. 


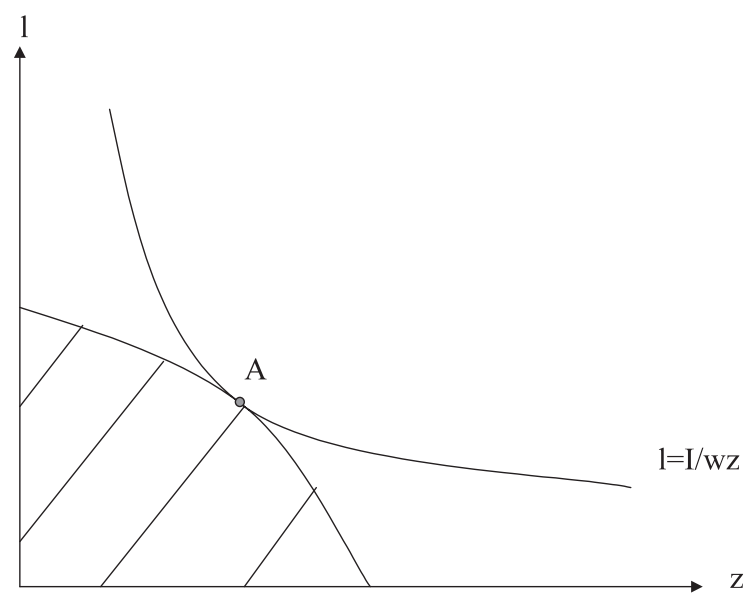

Fig. 1. The effort minimization problem.

The curve with equation $l=I / w z$ represents all the combinations of $(z, l)$ that yield a given level of lifetime income $I$. Note that the slope of this curve is given by $-I / w z^{2}=-l / z$. To maximize utility, it is necessary that this income level be produced so as to minimize the lifetime disutility of labor (effort). The shaded area represents the $(z, l)$ combinations that generate a level of effort lower than or equal to a fixed level $E$. The optimal (non-distorted) $(z, l)$ choice is given by the point $A$ satisfying

$$
\frac{R^{\prime}(z) / R(z)}{V^{\prime}(l) / V(l)}=\frac{l}{z}
$$

that is, where the marginal rate of substitution between $l$ and $z$ is equal to the slope of the lifetime earnings curve; this is of course exactly equivalent to condition (13). Rearranging the terms, one obtains:

$$
\varepsilon_{V}(l) \equiv \frac{l V^{\prime}(l)}{V(l)}=\frac{z R^{\prime}(z)}{R(z)} \equiv \varepsilon_{R}(z)
$$

which corresponds to an equality between the elasticities of disutility for the work week $\varepsilon_{V}(l)$ and that for the retirement age $\varepsilon_{R}(z)$. For simplicity, we shall refer to the first one as the "work week elasticity" and to the second one as the "retirement elasticity".

We assume the two following monotonicity properties:

Assumption 1. $\varepsilon_{V}(l)$ and $\varepsilon_{R_{j}}(z)(j=h, l)$ are non-decreasing functions (of $l$ and $z$, respectively). ${ }^{5}$

\footnotetext{
5 A necessary and sufficient condition for this is that: (i) $1-\left(l V^{\prime}(l)\right) /(V(l))+\left(l V^{\prime \prime}(l)\right) /\left(V^{\prime}(l)\right)>0$ for every $(l)$; (ii) $1-\left(z R^{\prime}(z)\right) /(R(z))+\left(z R^{\prime \prime}(z)\right) /\left(R^{\prime}(z)\right)>0$ for every $z$.
} 
Assumption 2. For every $z$, one has $\varepsilon_{R_{l}}(z) \leq \varepsilon_{R_{h}}(z)$. In words, for any given age of retirement, the retirement elasticity of the more disabled individual is greater than or equal to the retirement elasticity of the more healthy individuals.

These two assumptions allow us to compare the two optimal choices of $l$ and $z$ for the same aggregate earnings $I$ with two individuals differing respectively in their productivity and their preferences for retirement.

Let us start with the case where individuals differ solely in their productivity. In Fig. 2, the more able individual (individual 2) chooses both a lower $z$ and a lower $l$. This is the case if $\varepsilon_{R}(z)$ and $\varepsilon_{V}(l)$ are strictly increasing functions of $z$ and $l$. For the special cases where $R$ or $V$ are isoelastic functions, the choice of either $l$ (horizontal arrow) or $z$ (vertical arrow) are the same for the two individuals (see Eq. (15)). To sum up, Assumption 1 ensures that for a given level of lifetime earnings, the $(z, l)$ choice of an individual with a higher ability lies south west of the point chosen by a less able individual.

We now turn to the case where individuals solely differ in their health status, where Assumption 2 becomes relevant. Fig. 3 shows that the individual who has a greater disutility for the retirement age (individual 2) will choose a higher $l$ and a lower $z$ than the other individual if the marginal rate of substitution between $l$ and $z$ is higher for this individual, that is, if $\varepsilon_{R_{h}}(z)>\varepsilon_{R_{l}}(z)$. In the extreme example where $\varepsilon_{R_{h}}(z)=\varepsilon_{R_{l}}(z)$, the two isoeffort curves will be parallel in the $(z, l)$ space so that for the same aggregate earnings, they will choose the same pair $(z, l)$.

Before proceeding, let us revisit our specification of preferences and further comment on the type of heterogeneity which is implied. An individual's choices are crucially affected by his marginal rate of substitution between length of working life $z$ and work intensity $l$. As brought out by Eq. (13), this marginal rate of substitution is affected similarly by an increase in $R(z)$ as by a decrease in $V(l)$. This is consistent with our

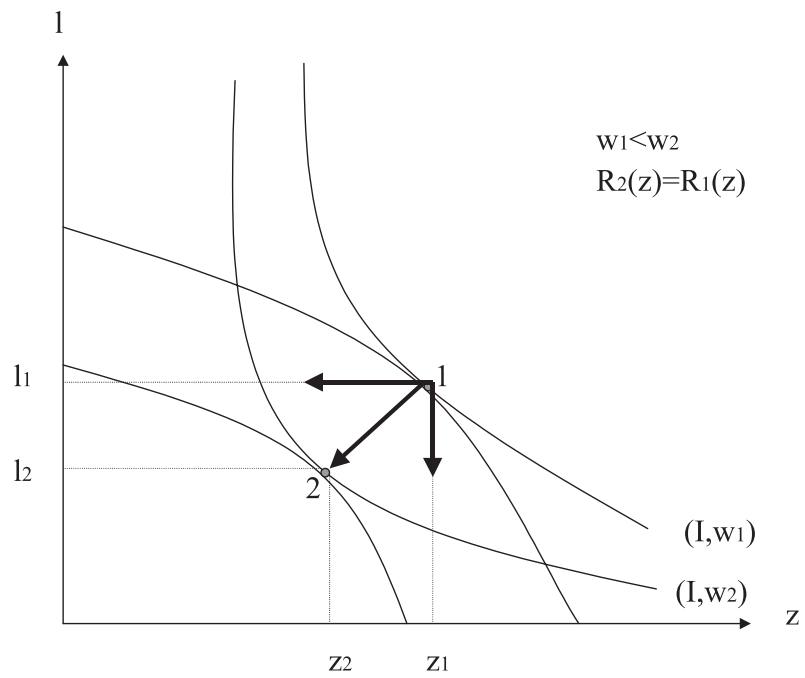

Fig. 2. Choices of $(z, l)$ for the two productivity types. 


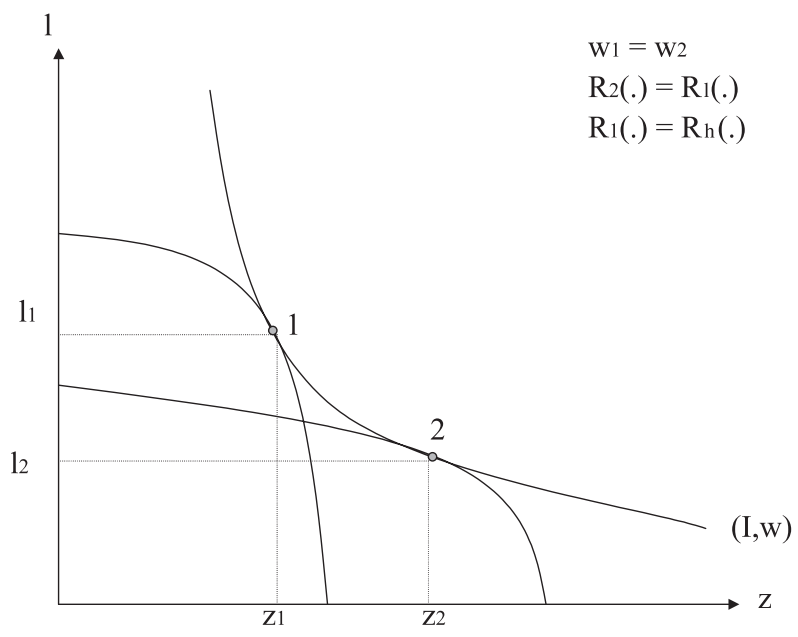

Fig. 3. Choices of $(z, l)$ for the two health types.

argument that the identical $V(l)$ assumption is not effectively restrictive. A more severe restriction is brought in by Assumption 2 according to which a higher $R(z)$ results in an increase in the ratio $z / l$ for a given level of lifetime income; see Fig. 3. Consequently, for a given level of $I=w l z$, an individual who is in poor health will prefer to work fewer years but work more intensely during his active life (otherwise $I$ could not be the same). An alternative view on health would have been to assume that to earn a given lifetime income individuals who are in poor health spread out effort over a longer period (they adopt a lower $l$ but a larger $z)^{6}$

\subsection{The social optimum}

The above market solution can be contrasted with the first-best social optimum, which is obtained when the social planner observes $w_{i}$ and $R_{j}$. We consider a utilitarian social welfare function given by $\Sigma_{i j} f_{i j} U_{i j}$, where $f_{i j}$ is the proportion of type $i j$ 's individuals and $U_{i j}=h u\left(c_{i j}\right)-V\left(l_{i j}\right) R_{j}\left(z_{i j}\right)$ is the lifetime utility of $i j$ individuals. ${ }^{7}$ Welfare maximization is subject to the resource constraint that aggregate consumption cannot exceed aggregate production. This problem is given by:

$$
\max _{c_{i j}, l_{i j}, z_{i j}} \sum_{i, j} f_{i j}\left[h u\left(c_{i j}\right)-V\left(l_{i j}\right) R_{j}\left(z_{i j}\right)\right]-\mu \sum_{i} f_{i j}\left(h c_{i j}-w_{i} l_{i j} z_{i j}\right),
$$

\footnotetext{
6 This would amount to reversing Assumption 2, which is used to study distortions in the $(z, l)$ space.

7 We consider the utilitarian case to keep the expressions as simple as possible. Our analysis can easily be generalized to the case where social welfare is a weighted sum of individual utilities. This would not affect our main results. However, some specific results may change and some assumptions on the weights may be necessary; see footnote 13 .
} 
where $\mu$ is the Lagrange multiplier associated to the resource constraint. The first order conditions for every $i, j$ are:

$$
\begin{aligned}
& u^{\prime}\left(c_{i j}\right)-\mu=0 \\
& V^{\prime}\left(l_{i j}\right) R_{j}\left(z_{i j}\right)-\mu w_{i} z_{i j}=0 \\
& V^{\prime}\left(l_{i j}\right) R_{j}^{\prime}\left(z_{i j}\right)-\mu w_{i} l_{i j}=0
\end{aligned}
$$

Combining these expressions, we find for every type $i, j$ the non-distorted tradeoffs described by Eqs. (11), (12) and (13). In addition, Eq. (17) requires identical consumption levels for all individuals. ${ }^{8}$

With the reduced form utility function (6), the time dimension is implicit. In the laissezfaire solution, there is implicitly saving during the working period: $\left(w_{i} l_{i j}-c_{i j}\right) z_{i j}$ which is used to finance consumption during retirement: $\left(h-z_{i j}\right) c_{i j}$; recall that we have assumed a zero interest rate. Consequently, the first-best allocation $c_{i j}^{*}, l_{i j}^{*}, z_{i j}^{*}$ can be decentralized through a social security scheme with a non-distortionary (lump-sum) contribution $\left(w_{i} l_{i j}^{*}-c_{i j}^{*}\right) z_{i j}^{*}$ and (lump-sum) social security benefits equal to $\left(h-z_{i j}^{*}\right) c_{i j}^{*}$. The number of hours $l_{i j}$ and the age of retirement $z_{i j}$ would be chosen optimally according to Eqs. (17) and (18) coinciding with the first-best tradeoffs.

The first-best solution and its decentralization have been derived under the assumption that individual types $w_{i}$ and $R_{j}$ are observable. When there is asymmetric information, first-best lump sum transfers are (generally) not feasible; redistribution then has to rely on potentially distortionary taxes and transfers based on observable variables. In the remainder of the paper, we adopt an information structure that is inspired by the optimal taxation literature. Specifically, we assume that productivities $w_{i}$, labor supply $l_{i j}$ and health status $R_{j}$ are not observable, while (weekly) before tax income $y_{i j}=l_{i j} w_{i}$ is observable. An added feature of our analysis compared to conventional optimal tax models is that the retirement age $z_{i j}$ is also observable. Taxes and transfers can then be based both on $y$ and on $z$. We use a non-linear tax function $T(y, z)$, which, as shown by Eq. (4), accounts for income taxation, payroll taxes and retirement benefits.

The assumption that productivity and health status cannot be observed by public authorities but are fully known by individuals is a strong though standard assumption. In fact, even with imperfect observability, our results would hold true as long as there is asymmetry of information. Also the assumption that individuals know their health status at the beginning could be a problem in a truly intertemporal model. However, here the multiperiod dimension is collapsed into a single period. We come back on this in Section 6.

\footnotetext{
8 When social welfare puts different weights on the individual utilities, the marginal social valuations of consumption, rather than the actual consumption levels, must be the same for all individuals.
} 


\section{Second-best taxation}

\subsection{Implementation}

Let us first examine how an individual's choices are affected by a non-linear income tax schedule $T(y, z)$. The first-order conditions of this modified individual problem are crucial for understanding the implementation of the optimal tax policy derived below. The individual's problem now becomes

$$
\begin{aligned}
& \max u\left(c_{i j}\right)-V\left(\frac{y_{i j}}{w_{i}}\right) R_{j}\left(z_{i j}\right) \\
& \text { s.t. } c_{i j}=y_{i j} z_{i j}-T\left(y_{i j}, z_{i j}\right)
\end{aligned}
$$

From the first order conditions, one obtains:

$$
\begin{aligned}
\operatorname{MRS}_{c l}^{i j} & =w_{i} z_{i j}\left(1-\frac{1}{z_{i j}} \frac{\partial T\left(y_{i j}, z_{i j}\right)}{\partial y_{i j}}\right) \\
\operatorname{MRS}_{c z}^{i j} & =y_{i j}\left(1-\frac{1}{y_{i j}} \frac{\partial T\left(y_{i j}, z_{i j}\right)}{\partial z_{i j}}\right)
\end{aligned}
$$

and the implicit relation between $l$ and $z$ being:

$$
\mathrm{MRS}_{l z}^{i j}=\frac{l_{i j}}{z_{i j}}\left[1-\theta_{i j}\right]
$$

where

$$
\theta_{i j}=\frac{T_{z}^{i j} / y_{i j}-T_{y}^{i j} / z_{i j}}{1-T_{y}^{i j} / z_{i j}}
$$

$\theta_{i j}$ is the marginal tax rate of $z$ with respect to $l$.

Distortions in the $(l, c)$ and $(z, c)$ choices are assessed by comparing Eqs. (20) and (21) to their laissez-faire counterparts Eqs. (11) and (12). Not surprisingly, a positive marginal tax on either $l$ or $z$ implies a downward distortion on the corresponding variable.

Let us now turn to the tradeoff between $z$ and $l$. Comparing Eq. (22) to its laissezfaire and first-best counterpart, Eq. (13) shows that, when $\theta_{i j}$ is equal to zero, there is no distortion (in the tradeoff between $z$ and $l$ ). This is true in particular when $T\left(y_{i j}, z_{i j}\right)=$ $T\left(y_{i j} z_{i j}\right)$, so that the tax depends only on total lifetime income. Furthermore, if $\theta_{i j}$ is negative $z$ is encouraged with respect to $l$, while a positive $\theta_{i j}$ implies a distortion in favor of $l{ }^{9}$

\footnotetext{
9 The distortions mentioned here are substitution effects, for given levels of $I$.
} 
An alternative view on these distortions consists in saying that the choice between $z$ and $l$ is distorted downwards if individuals who retire earlier pay less taxes, for a given level of before tax lifetime income $I$, that is, when: ${ }^{10}$

$$
\left.\frac{\mathrm{d} T\left(y_{i j}, z_{i j}\right)}{\mathrm{d} z_{i j}}\right|_{I}=\frac{\partial T\left(y_{i j}, z_{i j}\right)}{\partial z_{i j}}-\frac{y_{i j}}{z_{i j}} \frac{\partial T\left(y_{i j}, z_{i j}\right)}{\partial y_{i j}}>0
$$

Using Eq. (23), it appears that Eq. (24) amounts to $\theta_{i j}>0 .{ }^{11}$ Consequently, the two alternative ways to define the distortions are effectively equivalent.

\subsection{The second-best optimum}

To determine the second-best optimum, we concentrate on settings with two types only (each of which being characterized by a specific value for the two parameters of heterogeneity). We assume that the correlation between the two characteristics is non-positive. $^{12}$

We will first present the general case where the two types effectively differ in the two dimensions. Then, we consider two subcases where heterogeneity is only in one dimension. Subcase (a) will refer to the case where both individuals have the same preference over the age of retirement but differ in their productivity. Subcase (b) will refer to the case where both agents differ in their preference for the age of retirement but have the same productivity.

Formally, the economy is composed of two agents $2(=h l)$ and $1(=l h)$ being characterized respectively by a pair $\left(w_{2}=w_{h}, R_{2}(z)=R_{1}(z)\right)$ and $\left(w_{1}=w_{l}, R_{1}(z)=R_{h}(z)\right)$, with strict inequalities $w_{2}>w_{1}$ and $R_{2}(z)<R_{1}(z)$. In case (a), the two agents will have $R_{1}(z)=R_{2}(z)=R_{h}(z)$ and in case (b) $w_{1}=w_{2}=w_{h}$.

The problem of the government is directly obtained from Eq. (16) to which we add the incentive compatibility constraint that agent 2 does not want to mimic agent $1 .{ }^{13}$ This yields the following problem:

$$
\begin{aligned}
& \max _{c_{i}, y_{i}, z_{i}} \sum_{i} f_{i} U_{i}+\mu\left(\sum_{i} f_{i}\left(y_{i} z_{i}-h c_{i}\right)\right) \\
& \quad+\lambda\left(h u\left(c_{2}\right)-V\left(\frac{y_{2}}{w_{2}}\right) R_{2}\left(z_{2}\right)-h u\left(c_{1}\right)+V\left(\frac{y_{1}}{w_{2}}\right) R_{2}\left(z_{1}\right)\right)
\end{aligned}
$$

10 In the same way, there will be an upward distortion of the $(z, l)$ choice when:

\footnotetext{
$\left.\frac{\mathrm{d} T_{i j}\left(y_{i j}, z_{i j}\right)}{\mathrm{d} y_{i j}}\right|_{I}=\frac{\mathrm{d} T_{i j}\left(y_{i j}, z_{i j}\right)}{\mathrm{d} y_{i j}}-\frac{z_{i j}}{y_{i j}} \frac{\mathrm{d} T_{i j}\left(y_{i j}, z_{i j}\right)}{\mathrm{d} z_{i j}}>0$.

11 As long as $1-T_{y}^{i j} / z_{i j}>0$, a condition which necessarily holds at an interior solution; see Eq. (20).

12 We exclude the strict positive correlation case for which little can be said except when one difference overwhelmingly dominates the other.

13 In the utilitarian case (and with the considered configuration of types), this constraint is necessarily binding. However, when the social welfare function is a weighted sum of individual utilities and when the weight of the (able and/or healthy), type 2 is sufficiently large this may not be true anymore. Though formally possible, this does not appear to be a particular relevant case to consider. For the rest, as long as the constraint from 2 to 1 is binding, all our result in this section go through for a weighted social welfare function.
} 
where $\mu$ and $\lambda$ denote the multipliers associated with the revenue and the incentive compatibility constraints. ${ }^{14}$ First order conditions with respect to $c_{i}, y_{i}$ and $z_{i}$ :

$$
\begin{aligned}
& f_{1} u^{\prime}\left(c_{1}\right)-\mu f_{1}-\lambda u^{\prime}\left(c_{1}\right)=0 \\
& f_{2} u^{\prime}\left(c_{2}\right)-\mu f_{2}+\lambda u^{\prime}\left(c_{2}\right)=0 \\
& -\frac{f_{1}}{w_{1}} V^{\prime}\left(l_{1}\right) R_{1}\left(z_{1}\right)+\mu f_{1} z_{1}+\frac{\lambda}{w_{2}} V^{\prime}\left(\bar{l}_{2}\right) R_{2}\left(z_{1}\right)=0 \\
& -\frac{f_{2}}{w_{2}} V^{\prime}\left(l_{2}\right) R_{2}\left(z_{2}\right)+\mu f_{2} z_{2}-\frac{\lambda}{w_{2}} V^{\prime}\left(l_{2}\right) R_{2}\left(z_{2}\right)=0 \\
& -f_{1} V\left(l_{1}\right) R_{1}{ }^{\prime}\left(z_{1}\right)+\mu f_{1} y_{1}+\lambda V\left(\bar{l}_{2}\right) R_{2}^{\prime}\left(z_{1}\right)=0 \\
& -f_{2} V\left(l_{2}\right) R_{2}{ }^{\prime}\left(z_{2}\right)+\mu f_{2} y_{2}-\lambda V\left(l_{2}\right) R_{2}^{\prime}\left(z_{2}\right)=0
\end{aligned}
$$

where the upper bar denotes the choice of the mimicker, so that $\bar{l}_{2}=y_{1} / w_{2}$, i.e., the quantity of labor type 2 must supply to earn $y_{1}$.

Combining Eqs. (27), (29) and (31), one obtains non-distorted tradeoffs for type 2; marginal rates of substitution for this individual continue to be given by Eqs. (11)-(13). From Eqs. (20), (21) and (22), this implies that marginal tax rates with respect to $y$ and $z$ are zero so that we also have $\theta_{2}=0$. This is the usual no distortion at the top property.

We now turn to individual 1 and study successively his tradeoffs in the $\left(l_{1}, c_{1}\right),\left(z_{1}, c_{1}\right)$ and $\left(z_{1}, l_{1}\right)$ planes. This leads us to the determination of the marginal income tax rate and the marginal implicit tax on continued labor force participation, which apply to this individual. We can also study how (if at all) his tradeoff between weekly and lifetime labor supply is affected.

\subsubsection{Marginal income tax rate}

Eqs. (26) and (29) yield:

$$
\operatorname{MRS}_{c l}^{1}=\left[\frac{1-\frac{\lambda}{f_{1}}}{1-\frac{\lambda}{f_{1}} \frac{w_{1}}{w_{2}} \frac{\overline{\mathrm{MRS}_{c l}^{2}}}{\mathrm{MRS}_{c l}^{1}}}\right] w_{1} z_{1}
$$

where $\overline{\mathrm{MRS}}^{2}$ denotes individual 2's marginal rate of substitution when mimicking individual 1. We have $\operatorname{MRS}_{c l}^{1}>\overline{\operatorname{MRS}}_{c l}^{2}$ because $\bar{l}_{2}<l_{1}$ and $R_{2}\left(z_{1}\right)<R_{1}\left(z_{1}\right)$. Consequently, Eq. (32) implies $\mathrm{MRS}_{c l}^{1}<w_{1} z_{1}$ so that there is a marginal downward distortion in the work week. In other words, by Eq. (20), the marginal tax on weekly income, $y$, is positive. This property does not come as a surprise and it is in line with the standard property obtained in the optimal income tax literature.

\footnotetext{
14 When contrasting this with the first-best problem recall that index 2 stands for $h l$, while 1 stands for $l h$. Also note that we now optimize with respect to $y$ (observable variable) rather than $l$.
} 


\subsubsection{Marginal tax on continued labor force participation}

Now combining Eqs. (26) and (30), one obtains:

$$
\mathrm{MRS}_{c z}^{1}=\left[\frac{1-\frac{\lambda}{f_{1}}}{1-\frac{\lambda}{f_{1}} \frac{w_{1}}{w_{2}} \frac{\overline{\mathrm{MRS}_{c z}^{2}}}{c z}}\right] y_{1}
$$

where $\mathrm{MRS}_{c z}^{1}>\overline{\mathrm{MRS}}_{c z}^{2}$. Consequently, one has $\mathrm{MRS}_{c z}^{1}<\mathrm{y}_{1}$ so that there is a marginal downward distortion on $z_{1}$. That is, for a given weekly labor supply, the individual is induced to choose a lower $z$ relative to $c$ than he would do in a first-best setting. By Eq. (21), the marginal tax on the retirement age is positive. Intuitively, this property can be explained by the fact that type 1 individuals have steeper indifference curves at any given point in the $(z, c)$ space than type 2 individuals. This is because type 1 individuals must be compensated more to accept to work longer than the mimicking individual (they are less healthy and have a higher weekly labor supply). This implies that, starting from the firstbest tradeoff, a variation $\mathrm{d} z_{1}<0$ along with a variation $\mathrm{d} c_{1}=\left(\mathrm{MRS}_{c z}{ }^{1}\right) \mathrm{d} z_{1}$ has no (first-order) effect on the utility of type 1 , but it decreases the utility of type 2 mimicking type 1 . Consequently, the downward distortion in $z_{1}$ is a way to relax an otherwise binding selfselection constraint.

To interpret this result, it is useful to recall Eq. (5) which relates $\partial T / \partial z$ to the implicit tax that the pension system imposes on continued labor force participation. It thus appears that it is optimal to adopt a retirement system with a benefit formula which induces early retirement for the low ability (and high disutility) individual.

A remarkable feature of this result is that it holds irrespective of the exact structure of heterogeneity. ${ }^{15}$ To be more precise, the result obtains just as well for a case where individuals differ mainly (or even solely) in productivity as it holds for the case where they differ mainly (or exclusively) in health status.

\subsubsection{Weekly vs. lifetime labor supply}

Combining Eqs. (32) and (33), we find:

$$
\operatorname{MRS}_{l z}^{1}=\left[\frac{1-\frac{\lambda}{f_{1}} \frac{w_{1}}{w_{2}} \frac{\overline{\mathrm{MRS}}_{c l}^{2}}{\mathrm{MRS}_{c l}^{1}}}{1-\frac{\lambda}{f_{1}} \frac{\overline{M R S}_{c z}^{2}}{\mathrm{MRS}_{c z}^{1}}}\right] \frac{l_{1}}{z_{1}}
$$

\footnotetext{
15 As long as health and productivity are positively correlated so that incentive constraints bind from the healthy productive to the unhealthy low productivity individuals. Observe that the result can also be extended beyond two types, as long as the same patterns of binding incentive constraints arise. This is not a serious restriction when there is a one to one and positive relationship between health status and productivity. It is more problematic, though in a truly multidimensional setting; see Cremer et al. (2001) for a discussion.
} 
which, as shown in Appendix A, implies

$$
\operatorname{MRS}_{l z}^{1}=\frac{R_{1}^{\prime}\left(z_{1}\right) / R_{1}\left(z_{1}\right)}{V^{\prime}\left(l_{1}\right) / V\left(l_{1}\right)} \gtreqless \frac{l_{1}}{z_{1}} \Leftrightarrow \frac{\varepsilon_{V}\left(l_{1}\right)}{\varepsilon_{V}\left(\overline{l_{2}}\right)} \gtreqless \frac{\varepsilon_{R_{1}}\left(z_{1}\right)}{\varepsilon_{R_{2}}\left(z_{1}\right)} .
$$

Regarding the tradeoff between $l$ and $z$, we thus obtain an ambiguous result. Whether the $(z, l)$ is distorted upward or downward (i.e., whether $\theta_{1}$ is negative or positive) depends upon the relative differences in characteristics. If the ratio between week labor elasticities of individual 1 and the mimicker is larger than the one between their retirement elasticities, the $(z, l)$ choice is upward distorted $\left(\theta_{1}>0\right)$. Otherwise, it is downward distorted $\left(\theta_{1}<0\right)$. In order to better understand the role of the relative differences in the two characteristics, two extreme cases are now considered. Throughout this discussion, we have to keep in mind that we are talking here about relative distortions between $l$ and $z$; we know from the previous subsections that both of these variables are effectively distorted downward (relative to the numeraire good). Roughly speaking we are thus now determining which type of labor supply faces the most heavy distortion.

\subsubsection{Subcase (a): $R_{1}(z)=R_{2}(z)$}

In this case, individuals differ only in productivity. Consequently, we have $\varepsilon_{R_{1}}\left(z_{1}\right)=$ $\varepsilon_{R_{2}}\left(z_{1}\right)$ and Eq. (35) simplifies to:

$$
\operatorname{MRS}_{l z}^{1}=\frac{R_{1}^{\prime}\left(z_{1}\right) / R_{1}\left(z_{1}\right)}{V^{\prime}\left(l_{1}\right) / V\left(l_{1}\right)} \gtreqless \frac{l_{1}}{z_{1}} \Leftrightarrow \varepsilon_{V}\left(\overline{l_{2}}\right) \lesseqgtr \varepsilon_{V}\left(l_{1}\right) .
$$

First notice that, when $V$ is isoelastic, the $\left(z_{1}, l_{1}\right)$ choice is not distorted. As discussed earlier, if $V$ is isoelastic, $z$ is fixed and equal for both individuals. The weekly income is then distorted downwards while the age of retirement is the same as in the first-best.

In the general case, when $\varepsilon_{V}$ is increasing (Assumption 1), we have $\varepsilon_{V}\left(\overline{l_{2}}\right)<\varepsilon_{V}\left(l_{1}\right)$ yielding $\mathrm{MRS}_{l z}^{1}$, the marginal rate of substitution between $l$ and $z$ is greater in absolute value than the slope of the gross income curve. From Eq. (23), this is equivalent to $\theta_{1}<0$. Consequently, for a given $I$, individual 1 has, at the second-best, a greater $z$ and a lower $l$ relative to the first-best choice. ${ }^{16}$

This result is an interesting extension of the optimal income taxation literature. The intuition can be understood most easily by considering the slope of individual indifference curves in the $(z, y)$ space (i.e., the space of observable variables). When the elasticity of $v$ is increasing (in $l$ ), individual 2 prefers to have a relatively greater weekly income than individual 1 for a given gross life cycle income. Consequently, the slope of the iso-effort curve (indifference curve) is lower for individual 2 in the $(z, y)$ space; see Appendix B. To make type 1's consumption bundle less attractive to type 2 (and relax an otherwise binding incentive constraint), the optimal policy then implies a relatively higher retirement age and a lower weekly labor supply for individual $1 .^{17}$

16 But $I$ is of course not the same as in the first-best. Consequently, this property is not in contradiction to the fact that $z$ faces a positive marginal tax (and is distorted downward with regard to the numeraire).

17 The argument presented above for the $(z, c)$ space can easily be adapted to apply here. 


\subsubsection{Subcase (b): $w_{1}=w_{2}$}

Individuals now differ solely in their health status and Eq. (35) reduces to

$$
\operatorname{MRS}_{l z}^{1}=\frac{R_{1}^{\prime}\left(z_{1}\right) / R_{1}\left(z_{1}\right)}{V^{\prime}\left(l_{1}\right) / V\left(l_{1}\right)} \gtreqless \frac{l_{1}}{z_{1}} \Leftrightarrow \varepsilon_{R_{2}}\left(z_{1}\right) \gtreqless \varepsilon_{R_{1}}\left(z_{1}\right) .
$$

When $R_{1}$ and $R_{2}$ have the same elasticity (for any given level of $z$ ), there is no distortion for the $\left(z_{1}, l_{1}\right)$ choice. A simple example of this is when $R_{1}(z)=\delta R_{2}(z)$ with $\delta>1$. As shown previously, the $(z, l)$ choices are the same for the two individuals for a given gross life cycle income.

In the general case when $\varepsilon_{R_{2}}\left(z_{1}\right)<\varepsilon_{R_{1}}\left(z_{1}\right)$ (Assumption 2), we obtain $\mathrm{MRS}_{l z}^{1}<l_{1} / z_{1}$, so that the slope of the effort frontier is lower than the slope of the gross income line. Using Eq. (23), we thus obtain $\theta_{1}>0$. Consequently, for $I$ given, at the second-best, the individual will choose a greater $l$ and a lower $z$ relative to the first-best choice. In this special case, early retirement is encouraged.

This result is in contrast with that obtained in the previous subsection. This can be explained as follows. Individual 2 now prefers to have a (relatively) higher retirement age than individual 1 for a given gross life cycle income (the slope of the iso-effort curve is greater for individual 2 in the $(z, y)$ space; see Appendix B). Consequently, the incentive constraint can be relaxed by setting a relatively lower retirement age and a greater weekly labor supply for individual 1 .

\section{Numerical examples}

We now present some numerical examples with illustrative results for the three-types case, a setting not considered in the analytical part. These examples highlight the role of the relative differences in characteristics. They also incorporate the possibility of (costly) auditing for the health status as additional departure from the theoretical model. A fully fledged study of such controls goes beyond the scope of this paper. Our aim here is merely to provide an illustration, which provides some qualitative understanding of the impact of audits. It suggests that audits do not appear to represent a fundamental challenge to the main findings of this paper.

We use a quasi-linear utility function which implies the there are no income effects on labor supply. This leads to crisper results and facilitates their interpretation. However, it also imposes the restriction that everyone has the same marginal utility of income. To introduce concern for redistribution, we thus consider a social welfare function of the type $\sum_{i j} f_{i j} \Phi\left[U_{i j}\right]$, where $\Phi$ is a strictly concave function reflecting social preference for equity. The following specific functions are used:

$$
\begin{array}{ll}
\Phi(x)=x^{1-\rho} /(1-\rho), & u(c)=c, \\
V(l)=1 /(1-l)^{\beta}, & R_{j}(z)=1 /(1-z)^{\alpha_{j}}
\end{array}
$$

with fixed parameters $\beta=2, \rho=2$. Observe that $R$ is an increasing function of $\alpha$ so that a higher level of this parameter reflects a higher disutility of $z$ (and thus a lower health 
status). The distribution of characteristics is uniform. The three types are defined such that $w_{1}=w_{2}=200<w_{3}=400$ and $\alpha_{1}=\alpha_{h}=2>\alpha_{2}=\alpha_{3}=\alpha_{l} \in\{1.5,1.8,1.9\}$. In words, type 3 is productive (high wage) and healthy (low $\alpha$ ). Type 1 , on the other hand, is both less productive and less healthy. Finally, type 2 has a low productivity, but a high health status (same $\alpha$ as type 3 ).

The optimal allocations $(c, l$, and $z$ ), marginal tax rates on $z$ and $l$ and the relative marginal tax rates $\theta$ 's achieved for each of the three considered values of $\alpha_{l}$ are reported in Table 1 . We thus focus on variations in the health ratio $\alpha_{h} / \alpha_{l}$, which decreases from the first to the third scenario.

With the considered configuration, one may expect the self-selection constraint to go downwards from 3 to 2 (healthy and more productive mimicking healthy and less productive) and from 2 to 1 (healthy and less productive 22 mimicking unhealthy and less productive). However, our results indicate that the pattern of binding incentive constraints may be more complex than this conjecture would suggest.

When the health ratio is sufficiently large, the self-selection constraints go along the sequence $3-2-1$. Type 3 is subject to no distortion. Type $2-$ less productive than 3 but equally healthy - is subject to the same distortion as in subcase $(a)$ : downward distortion on $l$ relatively stronger than that on $z$. Type 1 is subject to the same distortion as in subcase (b): downward distortion on $z$ relatively stronger than that on $l$. The same pattern of results holds when the health ratio starts to decrease from $12 / 9$ to $10 / 9$. However, when it becomes sufficiently small, the incentive compatibility 23 constraint between type 3 and

Table 1

First- and second-best solution with three types of individuals with $w_{1}=w_{2}=200$ and $w_{3}=400$

\begin{tabular}{|c|c|c|c|c|c|c|c|}
\hline & & $c$ & 1 & $\mathrm{z}$ & $\mathrm{T}_{z}^{\prime}$ & $T_{y}^{\prime}$ & $\theta$ \\
\hline \multicolumn{8}{|c|}{$\alpha_{1}=2, \alpha_{2}=\alpha_{3}=1.5$} \\
\hline \multirow[t]{2}{*}{ Individual 1} & First-best & 96.90 & 0.551 & 0.551 & & & \\
\hline & Second-best & 84.95 & 0.545 & 0.535 & 12.67 & 0.042 & 0.040 \\
\hline \multirow[t]{2}{*}{ Individual 2} & First-best & 99.38 & 0.58 & 0.64 & & & \\
\hline & Second-best & 92.36 & 0.551 & 0.636 & 16.80 & 0.13 & -0.065 \\
\hline \multirow[t]{2}{*}{ Individual 3} & First-best & 122.42 & 0.64 & 0.70 & & & \\
\hline & Second-best & 133.92 & 0.64 & 0.70 & & & \\
\hline \multicolumn{8}{|c|}{$\alpha_{1}=2, \alpha_{2}=\alpha_{3}=1.8$} \\
\hline \multirow[t]{2}{*}{ Individual 1} & First-best & 88.72 & 0.551 & 0.551 & & & \\
\hline & Second-best & 81.07 & 0.549 & 0.545 & 4.94 & 0.015 & 0.016 \\
\hline \multirow[t]{2}{*}{ Individual 2} & First-best & 89.69 & 0.562 & 0.587 & & & \\
\hline & Second-best & 82.34 & 0.534 & 0.5761 & 14.97 & 0.11 & -0.064 \\
\hline \multirow[t]{2}{*}{ Individual 3} & First-best & 112.53 & 0.628 & 0.652 & & & \\
\hline & Second-best & 122.12 & 0.628 & 0.652 & & & \\
\hline \multicolumn{8}{|c|}{$\alpha_{1}=2, \alpha_{2}=\alpha_{3}=1.9$ (IC 31 binding) } \\
\hline \multirow[t]{2}{*}{ Individual 1} & First-best & 86.33 & 0.551 & 0.551 & & & \\
\hline & Second-best & 79.34 & 0.5443 & 0.546 & 5.58 & 0.032 & -0.008 \\
\hline \multirow[t]{2}{*}{ Individual 2} & First-best & 86.81 & 0.556 & 0.569 & & & \\
\hline & Second-best & 79.73 & 0.5351 & 0.56 & 11.85 & 0.086 & -0.051 \\
\hline \multirow[t]{2}{*}{ Individual 3} & First-best & 109.45 & 0.623 & 0.635 & & & \\
\hline & Second-best & 118.72 & 0.623 & 0.635 & 0 & 0 & 0 \\
\hline
\end{tabular}


Table 2

Second-best solution for a three-type case $\left(w_{1}=w_{2}=200, w_{3}=400\right.$ and $\left.\alpha_{1}=2, \alpha_{2}=\alpha_{3}=1.9\right)$ without and with auditing

\begin{tabular}{lllllllr}
\hline & & $c$ & \multicolumn{1}{c}{$\mathrm{c}$} & \multicolumn{1}{c}{$\mathrm{z}$} & \multicolumn{1}{c}{$\mathrm{T}_{z}^{\prime}$} & $T_{y}^{\prime}$ & $\theta$ \\
\hline Individual 1 & First-best & 86.3 & 0.551 & 0.551 & & & -0.009 \\
& SB (0) & 79.3 & 0.5444 & 0.546 & 5.58 & 0.032 & 0.001 \\
& SB (1) & 82.8 & 0.55 & 0.55 & 1.5 & 0.007 & 0.0006 \\
Individual 2 & SB (2) & 85 & 0.551 & 0.551 & 0.19 & 0.0006 & -0.051 \\
& First-best & 86.8 & 0.556 & 0.569 & & & -0.055 \\
& SB (0) & 79.7 & 0.535 & 0.56 & 11.85 & 0.086 & -0.052 \\
& SB (1) & 78.2 & 0.533 & 0.559 & 12.7 & 0.09 & 0.088 \\
Individual 3 & SB (2) & 77.4 & 0.534 & 0.56 & 12.15 & & \\
& First-best & 109.5 & 0.623 & 0.635 & & & \\
& SB (0) & 118.7 & 0.623 & 0.635 & & & \\
& SB (1) & 117.2 & 0.623 & 0.635 & & & \\
& SB (2) & 116.4 & 0.623 & 0.635 & & & ying \\
\hline
\end{tabular}

The scenarios are characterized by: (0) $k=15$, yielding $\pi=0$; (1) $k=5$, yielding $\pi=0.06$; and (2) $k=1$, yielding $\pi=0.11$. The incentive constraint $3-1$ is binding in scenarios (0) and (1) and not binding in (2).

type 1 becomes binding. In other words, both individuals 2 and 3 now have to be prevented from mimicking type 1 who benefits from an attractive early age of retirement. As a consequence, the marginal tax on type 1 individuals has to compromise between two binding incentive constraints. To be more precise, the incentive constraint $3-1$ pushes $\theta_{1}$ to be negative. This is because the disparity between $w_{3}$ and $w_{1}$ dominates the disparity between $\alpha_{3}$ and $\alpha_{1}$. The incentive constraint 2-1, on the other hand, pushes $\theta_{1}$ to be positive. As a consequence, the net distortion is ambiguous. For the parameter values, we reported it is negative.

In the next series of examples, we assume that $\alpha_{i}$ can be observed through a costly (perfect) audit. Productivity, however, remains unobservable and is not revealed by these audits. Auditing a fraction $\pi_{i}$ of individuals in group $i$ costs $f_{i} k \pi_{i}$, where $k$ is a positive constant. Individuals who are caught cheating are punished and have a utility level $U_{p}=0$, that is exogenous. Individuals who report to be in good health (type 2 or 3 ) will never be audited. ${ }^{18}$ We thus have $\pi_{2}=\pi_{3}=0$ and denote $\pi_{1}=\pi$. The solutions, including the optimal levels of $\pi$, are reported in Table 2 for three levels of $k .{ }^{19}$ Observe that the other parameter values correspond to the third scenario in Table 1 . When audit costs are high $(k=15)$, it is not optimal to audit at all and the solution is the same as in Table 1. For $k=5$ and $k=1$, there will be some auditing and it becomes less attractive to mimick type 1 individuals (to claim to be in bad health). Consequently, the incentive constraints towards type 1 are relaxed. This translates into a reduction of the distortions faced by this individual $\left(T_{z}^{\prime}\right.$ and $T_{y}^{\prime}$ decrease as $k$ decreases). Observe that when $k$ is small, the constraint $3-1$ ceases to be binding. Furthermore, audits also imply that the relative heterogeneity in $\alpha$ becomes less significant, which explains that the sign of $\theta_{1}$ is reversed.

Summing up, the results presented in Table 2 suggest that while the possibility of audit does bring in a number of additional interesting aspects, it does not appear to represent a

\footnotetext{
18 Since the audit does not reveal productivity, nothing can be gained by auditing individuals who report $\alpha_{1}$.

19 A formal statement of the problem, which is solved, is provided in Appendix C.
} 
fundamental challenge to the main findings of this paper. In particular, there continues to be a downward distortion on retirement age (except for the "top" individual). ${ }^{20}$

\section{Conclusion}

During the last decades, a number of European countries, some more than others, have expanded their social security systems in ways which have discouraged labor market participation in old age and thus fostered early retirement. We raised the question whether these disincentives to continued activity are necessarily the result of a bad tax-transfer scheme design. Can they instead be an ingredient of an optimally designed redistributive policy in a world of asymmetric information?

To address this issue, we have studied the design of retirement contributions and benefits in a setting where an optimal non-linear income tax is also available. Individuals differ in ability and/or health status (disutility of retirement age). Given the tax-transfer policy every individual chooses weekly labor supply (not observable) and retirement age (observable). As in the traditional income taxation literature, the optimal policy implies a positive marginal tax on the low ability (and/or less healthy) individual. More interestingly, the retirement benefit formula also introduces a bias towards early retirement in this individual's life cycle labor supply decisions. This distortion arises whatever the dominant source of heterogeneity (productivity or health). Finally, the relative distortion between weekly labor supply and retirement age (length of active life), if any, depends on the relative heterogeneity in ability and health.

This paper has some rather ambitious features. It aims at dealing with the question of disability, early retirement and regular retirement within the same model with individuals differing in both productivity and health. To pursue this ambition, we admittedly had to simplify other aspects of the model. Two of the key assumptions are that of constant (though endogenous) labor supply during the active life and that of no liquidity constraint. These two restrictions allow us to reduce an otherwise dynamic model into a static one.

Even though our paper deals in part with the issue of disability, its main focus is not disability insurance such as studied by Diamond and Mirrlees, 1978; 1986. The difference is most easily shown for the case where people only differ in health status. Then considering that individuals ex ante do not know their $R_{j}$, our social security scheme can be viewed as a one period disability insurance. The healthier a worker is (lower $R_{j}$ ) the later he will retire and also the less he will receive as net benefits $\left(-T_{j}\right)$. In Diamond and Mirrlees, all individuals are also identical ex ante but as time goes on they discover whether or not they are disabled. Truthtelling here implies that net benefits increase with the age of retirement. But clearly, this positive relation between benefit and retirement age is not the same as the negative relation one might observe in our one period model.

\footnotetext{
20 When $\pi=1$, the incentive constraint towards type 1 becomes irrelevant and the distortion disappears. As usual in audit problems, it can, however, be shown quite easily that, for $k>0$ and however small, it is never optimal to audit all individuals who report to be in bad health.
} 
We assume that not just productivity but also health (capacity to work long) are not observable. In the standard literature on disability, health is also not directly observable. However, some authors introduce the possibility of control, which at some cost reveals the health status; see, e.g., Diamond and Sheshinski (1995). Audit on health conditions can be introduced in our model and this will be the subject of a sequel to this paper. In the current paper, we have restricted ourselves to providing a few illustrative examples in Section 5. While the possibility of audit does bring in a number of additional interesting aspects, it does not appear to represent a fundamental challenge to the main findings of this paper. In particular, one can also expect that the optimal policy will continue to induce early retirement. As for the specific policy implications, one can conjecture that thanks to such audits early retirement benefits that are implicit to our optimal scheme will be higher than when audits are not available.

Another possible and natural extension is to allow for some health spending to correct for a high $R_{j}$. In other words, the health status would continue to have an exogenous (adverse selection) component, but it would also be affected by some specific expenditure, which may or may not be observable. In particular public provision of such a private good could be used as an additional instrument along with our social security scheme. (See on this Cremer and Gavahri, 1997.) The underlying argument here would be to reduce the incidence of disability rather than simply redistributing towards the less able. One can conjecture that if this instrument can contribute to narrow the gap between $R_{h}$ and $R_{l}$ its availability would lead to a welfare improvement.

Alternative specifications could lead to different outcomes. In particular, we deliberately assume that the utilitarian social planner takes into account differences in utilities without trying to correct them. In other words, it takes $R_{j}$ as a health parameter and not as a taste parameter. People with $R_{h}$ are unhealthy and ought to be compensated for that. If $R_{j}$ were viewed as taste for leisure, then it would make sense to launder out differences in $R_{j}$. (See on this Boadway et al., in press.).

With laundering out the result would change with tax inducement towards postponed activity for the $R_{h}$ 's workers now considered as "lazy". In the same line, we could have assumed some myopic intertemporal preferences leading to overly early retirement. Again, if the social planner had less myopic time preferences, the tax design could have been different.

Allowing for an explicit account of the time structure is clearly a priority on our research agenda. It is, however, a challenging objective in a setting with two, albeit correlated, factors of heterogeneity. Currently, there exists little work even on the separate issues of either health or productivity. On the health issue, there are naturally the papers by Diamond and Mirrlees (1978, 1986), which demonstrate the analytical difficulty of the question at hand. On the productivity issues, Britto et al. (1991) have shown how difficult is the issue of optimal non-linear income tax in a multiperiod setting. In view of these difficulties, it should not be surprising that the design an optimal tax-transfer scheme with intensive and extensive labor supply choices in a dynamic setting and with two characteristics is a formidable task. Our current paper clearly falls short of accomplishing this task. It is however a step in that direction which points at possible avenues for tackling more ambitious settings. 


\section{Acknowledgements}

This paper has been presented at the NBER-IFS TAPES "Conference on income taxation". We thank all the participants and particularly our discussants Antonio Rangel and Orazio Attanasio for helpful comments and suggestions. We are also grateful for the comments of the two referees and of the editor, Richard Blundel.

\section{Appendix A. Derivation of expression (35)}

Rearranging Eq. (34) yields

$$
\operatorname{MRS}_{l z}^{1}=\left[\frac{1-\frac{\lambda}{n_{1}} \frac{w_{1}}{w_{2}} \frac{\overline{M R S}_{c l}^{2}}{\mathrm{MRS}_{c l}^{1}}}{1-\frac{\lambda}{n_{1}} \frac{\overline{M R S}_{c z}^{2}}{\mathrm{MRS}_{c z}^{1}}}\right] \frac{l_{1}}{z_{1}}
$$

Consequently,

$$
\mathrm{MRS}_{l z}^{1} \gtreqless \frac{l_{1}}{z_{1}} \Leftrightarrow \frac{\overline{\operatorname{MRS}}_{c z}^{2}}{\operatorname{MRS}_{c z}^{1}} \gtreqless \frac{w_{1}}{w_{2}} \frac{\overline{M R S}_{c l}^{2}}{\mathrm{MRS}_{c l}^{1}}
$$

Using the definition of $\mathrm{MRS}_{c z}{ }^{1}$ and $\mathrm{MRS}_{c l}^{1}$ the property $w_{1}=w_{2} \overline{l_{2}} / l_{1}$ and rearranging yields

$$
\operatorname{MRS}_{l z}^{1} \gtreqless \frac{l_{1}}{z_{1}} \Leftrightarrow \frac{\frac{l_{1} V^{\prime}\left(l_{1}\right)}{V\left(l_{1}\right)}}{\frac{\overline{l_{2}} V^{\prime}\left(\overline{l_{2}}\right)}{V\left(l_{2}\right)}} \gtreqless \frac{\frac{R_{1}{ }^{\prime}\left(z_{1}\right)}{R_{1}\left(z_{1}\right)}}{\frac{R_{2}^{\prime}\left(z_{1}\right)}{R_{2}\left(z_{1}\right)}} .
$$

Multiplying the numerator and the denominator of the right hand side by $z_{1}$ then yields Eq. (35).

\section{Appendix B. Marginal rates of substitution in the $(z, y)$ space}

We compare the types marginal rates of substitution between $y$ at $z$ at any given point $(z, y)$. By definition, one has:

$$
\operatorname{MRS}_{y z}^{i j}(y, z)=\frac{V\left(\frac{y}{w_{i}}\right) R_{j}^{\prime}(z)}{\frac{1}{w_{i}} V^{\prime}\left(\frac{y}{w_{i}}\right) R_{j}(z)}
$$

Multiplying and dividing by $y z$ yields:

$$
\operatorname{MRS}_{y z}^{i j}(y, z)=\frac{y}{z} \frac{V\left(\frac{y}{w_{i}}\right)}{\frac{y}{w_{i}} V^{\prime}\left(\frac{y}{w_{i}}\right)} \frac{z R_{j}^{\prime}(z)}{R_{j}(z)}=\frac{y}{z} \frac{\varepsilon R_{j}(z)}{\varepsilon_{V}\left(\frac{y}{w_{i}}\right)}
$$


Assumption 1 implies $\varepsilon_{V}\left(\frac{y}{w_{h}}\right) \leq \varepsilon_{V}\left(\frac{y}{w_{l}}\right)$, so that

$$
\operatorname{MRS}_{y z}^{h j}(y, z) \geqslant \operatorname{MRS}_{y z}^{l j}(y, z) \text { for every } j=h, l .
$$

Consequently, in subcase (a), we have

$$
\operatorname{MRS}_{y z}^{2}(y, z) \geqslant \operatorname{MRS}_{y z}^{1}(y, z)
$$

Similarly, Assumption 2 implies $\varepsilon_{R_{l}}(z) \leq \varepsilon_{R_{h}}(z)$, so that

$$
\operatorname{MRS}_{y z}^{i h}(y, z) \geqslant \operatorname{MRS}_{y z}^{i l}(y, z) \text { for every } i=h, l .
$$

In subcase (b), we thus have

$$
\operatorname{MRS}_{y z}^{2}(y, z) \leq \operatorname{MRS}_{y z}^{1}(y, z) .
$$

In the more general case of negative correlation between the two types, one has

$$
\operatorname{MRS}_{y z}^{h l}(y, z) \gtreqless \operatorname{MRS}_{y z}^{l h}(y, z) \text { if and only if } \frac{\varepsilon_{R_{1}}(z)}{\varepsilon_{V}\left(\frac{y}{w_{h}}\right)} \gtreqless \frac{\varepsilon_{R_{h}}(z)}{\varepsilon_{V}\left(\frac{y}{w_{l}}\right)} \text {. }
$$

\section{Appendix C. The problem with three types and with auditing for the health status}

We provide a formal statement of the problem solved numerically in Section 5. We have three type of individuals with $w_{1}=w_{2}<w_{3}$ and $\alpha_{1}>\alpha_{2}=\alpha_{3}$. An audit reveals only $\alpha$ and thus identifies type 1 individuals. Let $\lambda_{i j}$ denote the incentive constraint from type $i$ to type $j(i, j=1,2,3, i \neq j)$. The Lagrangian expression of the government's problem is then given by

$$
\begin{aligned}
L= & f_{3}\left[u\left(c_{3}\right)-V\left(\frac{y_{3}}{w_{3}}\right) R_{3}\left(z_{3}\right)\right]+f_{2}\left[u\left(c_{2}\right)-V\left(\frac{y_{2}}{w_{2}}\right) R_{2}\left(z_{2}\right)\right] \\
& +f_{1}\left[u\left(c_{1}\right)-V\left(\frac{y_{1}}{w_{1}}\right) R_{1}\left(z_{1}\right)\right]+\mu\left[f_{1}\left(y_{1} z_{1}-c_{1}\right)+f_{2}\left(y_{2} z_{2}-c_{2}\right)\right. \\
& \left.+f_{3}\left(y_{3} z_{3}-c_{3}\right)-f_{1} k \pi\right]+\lambda_{32}\left(u\left(c_{3}\right)-V\left(\frac{y_{3}}{w_{3}}\right) R_{3}\left(z_{3}\right)-u\left(c_{2}\right)\right. \\
& \left.\left.+V\left(\frac{y_{2}}{w_{2}}\right) R_{2}\left(z_{2}\right)\right]\right)+\lambda_{31}\left(u\left(c_{3}\right)-V\left(\frac{y_{3}}{w_{3}}\right) R_{3}\left(z_{3}\right)-(1-\pi)\right. \\
& \left.\times\left[u\left(c_{1}\right)-V\left(\frac{y_{1}}{w_{3}}\right) R_{3}\left(z_{1}\right)\right]-\pi U^{p}\right)+\lambda_{21}\left(u\left(c_{2}\right)-V\left(\frac{y_{2}}{w_{2}}\right) R_{2}\left(z_{2}\right)\right. \\
& \left.-(1-\pi)\left[u\left(c_{1}\right)-V\left(\frac{y_{1}}{w_{1}}\right) R_{2}\left(z_{1}\right)\right]-\pi U^{p}\right),
\end{aligned}
$$

where we have assumed that upwards incentive constraint are not binding so that $\lambda_{23}=\lambda_{13}=\lambda_{12}=0$. Setting $\pi=0$ in this expression yields the problem without auditing (solved in Table 1). To solve the problem with auditing, one also optimizes with respect to $\pi$. Observe that the incentive constraint 3-2 is standard and corresponds to that in Eq. 
(25). The same is true for the other constraints when $\pi=0$. However, when $\pi>0$, the utility of a mimicker is a random variable.

\section{References}

Blondal, S., Scarpetta, S., 1998a. Falling Participation Rates Among Older Workers in the OECD Countries. OECD, Paris.

Blondal, S., Scarpetta, S., 1998b. The retirement decision in OECD countries. OECD-EDWP 202, 1-76.

Boadway, R., Marchand, M., Pestieau, P., del M. Racionero, M., 2002. Optimal redistribution with heterogeneous preferences for leisure. Journal of Public Economic Theory 4, 475-498.

Britto, D., Hamilton, J., Stiglitz, J., Slutsky, S., 1991. Dynamic optimal income taxation with government commitment. Journal of Public Economics 44, 15-35.

Crawford, V.P., Lilien, D.M., 1981. Social security and the retirement decision. Quarterly Journal of Economics $95,505-529$.

Cremer, H., Gavahri, F., 1997. In-kind transfers, self-selection and optimal tax policy. European Economic Review 41, 97-114.

Cremer, H., Pestieau, P., Rochet, J.-Ch., 2001. Direct versus indirect taxation. The design of the tax structure revisited. International Economic Review 42, 781-799.

Diamond, P., Mirrlees, J., 1978. A model of social insurance with variable retirement. Journal of Public Economics $10,295-326$.

Diamond, P., Mirrlees, J., 1986. Payroll tax financed social insurance with variable retirement. Scandinavian Journal of Economics 88, 25-50.

Diamond, P., Sheshinski, E., 1995. Economic aspects of disability benefits. Journal of Public Economics 57, 123.

Fabel, D., 1994. The Economics of Pension and Variable Retirement Schemes Wiley, New York.

Gruber, G., Wise, D., 1999. Social Security and Retirement Around the World The Chicago Univ. Press, Chicago.

Lazear, E.P., 1979. Why is there mandatory retirement? Journal of Political Economy 87, 1261-1269.

Lumsdaire, R.L., Mitchell, D., 1999. New developments in the economic analysis of retirement. In: Ashenfelter, O., Card, D. (Eds.), Handbook of Labor Economics, vol. 3. North Holland, Amsterdam, pp. $3261-3307$.

Maderner, N., Rochet, J.-C., 1995. Is it legitimate to encourage work sharing? Scandinavian Journal of Economics, 621-633.

Mirlees, J.A., 1971. An exploration of the theory of optimal taxation. The Review of Economics Studies 38, $175-208$. 